\title{
Inversionsbasierte optimale Steuerung von Batch-Kristallisationsprozessen zur Enantiomertrennung
}

\author{
Inversion-based Optimal Control of Batch Preferential Crystallization Processes
}

\begin{abstract}
Zusammenfassung: In diesem Beitrag wird zunächst eine inversionsbasierte Steuerung zur Realisierung vorgegebener Kristallgrößenverteilungen beim simultanen Batchbetrieb der bevorzugten Kristallisation in zwei gekoppelten Gefäßen unter symmetrischen Bedingungen behandelt. Anschließend wird ein Verfahren zur Minimierung der Batchdauer bei gegebener Reinheit und Produktmenge vorgestellt, welches auf der orbitellen Flachheit eines idealisierten Modells basiert. Die vorgeschlagene Methode wird anhand eines Beispiels mittels eines rechenintensiveren Verfahrens überprüft.
\end{abstract}

Schlüsselwörter: Enantiomere, Bevorzugte Kristallisation, Orbitelle Flachheit, Inversionsbasierte Steuerung, Optimale Steuerung.

\begin{abstract}
In this paper, inversion-based control for the realization of desired crystal size distributions in the context of simultaneous batch preferential crystallization in two coupled vessels is discussed for symmetric conditions. Then, the problem of minimizing batch time under given constraints regarding product purity and mass is considered. It can be efficiently solved using the orbital flatness property of an idealized model. In an example, the proposed method is validated using a computationally more intensive approach.
\end{abstract}

Keywords: Enantiomers, Preferential Crystallization, Orbital Flatness, Inversion-based Control, Optimal Control.

\footnotetext{
*Korrespondenzautor: Steffen Hofmann, Technische Universität Berlin, E-Mail: hofmann@control.tu-berlin.de

Jörg Raisch: Technische Universität Berlin und Max-Planck-Institut für Dynamik komplexer technischer Systeme
}

\section{Einleitung}

Enantiomere sind Moleküle mit spiegelbildlichem räumlichen Aufbau. Sie besitzen größtenteils identische physikalische und chemische Eigenschaften, können aber beispielsweise im menschlichen Körper sehr unterschiedliche Auswirkungen haben. Die Trennung von Enantiomeren ist deswegen insbesondere in der pharmazeutischen Industrie von großer Wichtigkeit. Die „Bevorzugte Kristallisation“ stellt eine attraktive Möglichkeit hierfür dar. Dieser Artikel gehört zu einer Reihe von Arbeiten, die sich auf den Batchbetrieb des Prozesses und die Klasse der konglomeratbildenden Enantiomere konzentrieren. Für diese Klasse kann durch Zugabe von Saatkristallen der bevorzugten (d.h. gewünschten) Enantiomersorte (z. B. $E_{1}$ ) zu einer razemischen (d.h. gleiche Massenanteile der beiden Enantiomersorten $E_{1}$ und $E_{2}$ aufweisende) Lösung und Betrieb in einem Arbeitsbereich, in dem die Sekundärnukleation die Primärnukleation dominiert, diese Enantiomersorte auskristallisiert und eine unzulässige Verunreinigung durch die Gegenenantiomersorte (d.h. unerwünschte Enantiomersorte) vermieden werden. Insbesondere wird im Folgenden eine Prozessvariante untersucht, die eine signifikante Effizienzsteigerung gegenüber dem Einzelbatch verspricht. Im so genannten simultanen Batchbetrieb, z.B. [7, 8], siehe Abbildung 1, erfolgt die Kristallisation beider Enantiomere gleichzeitig in zwei Gefäßen $A$ und $B$, zwischen denen Flüssigkeit ausgetauscht wird; der Austausch von Kristallen kann durch geeignete Filter ausgeschlossen werden. Vereinfacht gesprochen kann so die Bildung von Gegenenantiomer-Kristallen hinausgezögert werden.

Während die Steuerung, Regelung und Optimierung von Kristallisationsprozessen schon seit geraumer Zeit ein aktives Forschungsgebiet bilden (z. B., [5, 14, 18, 19, 24, $27,28]$ ), werden diese Fragestellungen in der jüngeren Zeit 


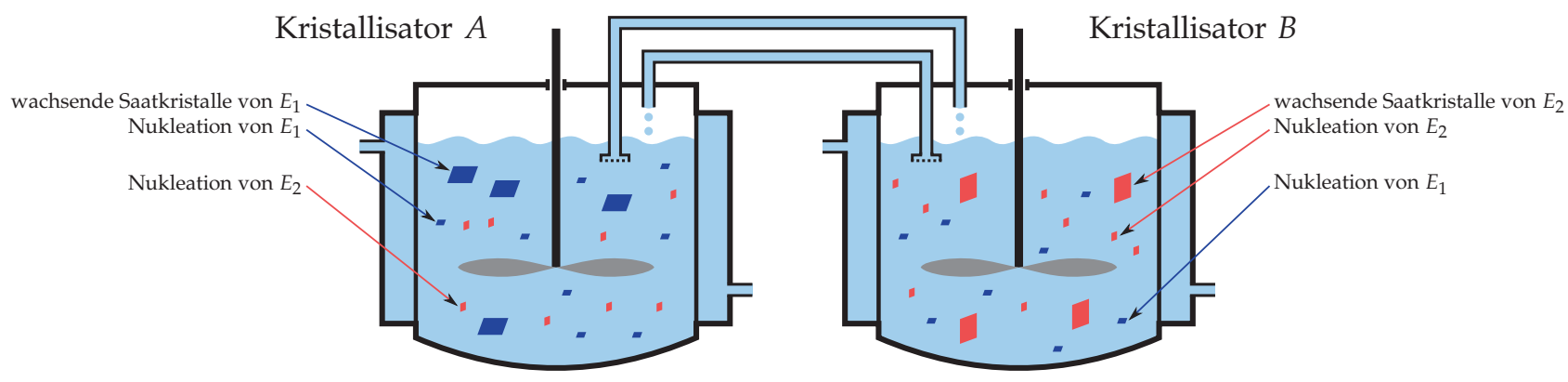

Abbildung 1: Simultane bevorzugte Kristallisation in zwei gekoppelten Gefäßen.

auch im Kontext der bevorzugten Kristallisation verstärkt untersucht, z. B. [1, 3, 13, 15, 16, 29]. In [1] wird ein Temperaturprofil zur Maximierung der kristallisierten Menge des bevorzugten Enantiomers unter Beachtung der zulässigen Verunreinigung bestimmt. In [3] wird der Prozess unter Berücksichtigung konkurrierender Ziele optimiert. Der vorliegende Aufsatz baut auf Vorarbeiten $[15,16]$ auf, die Verfahren zur dynamischen Inversion von Batchprozessen der bevorzugten Kristallisation behandeln, um mittels geeigneter Stellgrößenprofile vorgegebene Kristallgrößenverteilungen (KGVen) zu realisieren. Dabei müssen Differenzialgleichungen numerisch gelöst werden. Mit der dynamischen Inversion von Systemen beschäftigen sich beispielsweise auch $[6,10,11]$. In [16] wurde angedeutet, dass es bei dem simultanen Batchbetrieb zumindest unter der Annahme idealisierter Verhältnisse möglich ist, über die Vorgabe von KGVen für die Gegenenantiomere wichtige, beide Enantiomersorten betreffende Produkteigenschaften bzgl. Reinheit und Menge zu steuern bzw. zu optimieren. Gleichzeitig lässt sich - da die Modelle unter diesen idealisierenden Annahmen orbitell flach sind - die dynamische Inversion sehr effizient, d.h. ohne das numerische Lösen von Differenzialgleichungen durchführen. Es wurde auch festgestellt, dass die nicht ideale gegenseitige Durchmischung der Flüssigkeiten in beiden Gefäßen sowie ggf. übermäßige Nukleation der bevorzugten Enantiomere (in Relation zum Saatkristallwachstum) einen kritischen Einfluss auf die Eignung der Idealisierungen haben.

Für die folgenden Untersuchungen werden vollständig symmetrische Verhältnisse vorausgesetzt. Dies bedeutet, dass die für die simultane Kristallisation verwendeten Gefäße $A$ und $B$ identisch sind, die Prozessparameter und Anfangsbedingungen für $E_{1}$ in Gefäß $A$ und $E_{2}$ in Gefäß $B$ übereinstimmen, und die Prozessparameter und Anfangsbedingungen für $E_{2}$ in Gefäß $A$ und $E_{1}$ in Gefäß $B$ übereinstimmen. Außerdem wird von Störungen abgesehen. Folglich laufen bei Verwendung identischer Stellgrößenprofile alle Vorgänge in Kristallisator $B$ genau spiegelbildlich zu denen in Kristallisator $A$ ab. Dies ermöglicht eine kom- pakte Darstellung des Modells der gekoppelten Kristallisatoren, indem sich sämtliche Vorgänge durch ein geschlossenes System von partiellen und/oder gewöhnlichen Differenzialgleichungen (DGLen) für einen der beiden Kristallisatoren (z. B. A) beschreiben lassen. Die genannten Voraussetzungen sind natürlich in der Praxis nicht immer erfüllt, und werden deshalb in [16] als Idealisierung gekennzeichnet, deren Einfluss auf die erzielten Ergebnisse explizit untersucht wird. An dieser Stelle sollen symmetrische Verhältnisse jedoch als gegeben angenommen werden, und statt dessen soll die Eignung anderer, zunächst als kritischer eingestufter Idealisierungen untersucht werden, die eine wichtige Rolle bei der Lösungsfindung in den folgenden Abschnitten einnehmen.

Der Aufsatz ist wie folgt strukturiert: In Abschnitt 2 wird auf die Ein- und Ausgangsgrößen des Prozesses eingegangen und ein Modell bestehend aus partiellen Differenzialgleichungen sowie ein Modell bestehend aus gewöhnlichen Differenzialgleichungen angegeben. In Abschnitt 3 wird in Anlehnung an $[15,16]$ eine inversionsbasierte Steuerung sowohl auf Basis des detaillierten als auch eines approximativen Modells mit dem Ziel, eine gewünschte End-KGV zu realisieren, vorgestellt. In Abschnitt 4 wird darauf aufbauend eine zeitoptimale Steuerung berechnet und anhand eines Rechenbeispiels mit der direkten Optimierung der Stützpunkte des diskretisierten Stellgrößenprofils verglichen.

\section{Modell}

Man beachte das in Abbildung 1 dargestellte Schema zur simultanen bevorzugten Kristallisation in den Gefäßen $A$ und $B$, zwischen denen Lösung (jedoch keine Kristalle) ausgetauscht wird. Die bevorzugte Enantiomersorte in dem jeweiligen Kristallisator $\left(E_{1}\right.$ in Kristallisator $A, E_{2}$ in Kristallisator $B$ ) wird im Folgenden mit $p$ (preferred enantiomer) bezeichnet, während die Gegenenantiomersorte mit $c$ (counter enantiomer) bezeichnet wird. Bei Batchbe- 
ginn $(t=0)$ werden jeweils Saatkristalle für $p$, nicht aber für $c$, zugegeben.

Als Stellgröße wird bei Kristallisationsprozessen oft die Temperatur (der Suspension) $T$ angesehen. In der Tat kann diese i. A. so schnell und so genau eingestellt werden, dass diese Annahme gerechtfertigt ist. Im Folgenden werden zwei Modellierungsansätze im Zeitbereich gezeigt. Im ersten Fall ist der Systemzustand unendlichdimensional und beschreibt die kontinuierlichen Verteilungen der Kristallgrößen (Feststoffphase) und die gelösten Massen (Flüssigphase); durch eine Modellreduktion gelangt man zum zweiten Fall: Die Entwicklung der gelösten Massen und bestimmter integraler KGV-Größen lässt sich exakt durch ein System gewöhnlicher DGLen erster Ordnung beschreiben. Eine Reihe von die Flüssig- oder (kristalline) Feststoffphase beschreibenden Größen kommen als Ausgangs- bzw. Regelgrößen in Betracht. Beispielsweise können die gelösten Massen oder die Kristallmassen von Interesse sein, oder der (ggf. nicht direkt messbare) Modellzustand bzw. ein Teil davon soll z. B. zu einem vorgegebenen Ziel überführt werden (vgl. Abschnitt 3). Es existieren eine Reihe von Messverfahren für die Flüssigwie auch die Feststoffphase. Im Folgenden werden jedoch Steuerungen entworfen und Messungen spielen keine Rolle. Auch wird von Störungen (z. B. die Umgebungstemperatur oder Staubpartikel, die Kristallisationskeime darstellen) abgesehen.

\subsection{Modellstruktur}

Wenn nur Kristallwachstum und Nukleation berücksichtigt werden, und außerdem von kristallgrößenunabhängigem Wachstum ausgegangen wird, lässt sich, wie bei der Modellierung von Kristallisationssystemen üblich (siehe z.B. [20, 21]), die Evolution der Kristallpopulationen wie folgt über ein System aus partiellen DGLen (die sog. Populationsbilanzgleichungen, PBGen) beschreiben, welche über Nukleations- und Wachstumskinetiken miteinander sowie mit die Flüssigphasen beschreibenden gewöhnlichen DGLen gekoppelt sind. Für die beiden Enantiomersorten $p$ und $c$ in einem Kristallisator erhält man die PBGen:

$$
\begin{aligned}
& \frac{\partial f_{p}(L, t)}{\partial t}=-G_{p}(t) \frac{\partial f_{p}(L, t)}{\partial L}, \\
& \frac{\partial f_{c}(L, t)}{\partial t}=-G_{c}(t) \frac{\partial f_{c}(L, t)}{\partial L},
\end{aligned}
$$

mit den Rand- und Anfangsbedingungen

$$
f_{p}(0, t)=\frac{B_{p}(t)}{G_{p}(t)}, f_{p}(L, 0)=f_{p, \text { seed }}(L),
$$

$$
f_{c}(0, t)=\frac{B_{c}(t)}{G_{c}(t)}, f_{c}(L, 0) \equiv 0,
$$

wobei $t$ dieZeit repräsentiert, und die Größe eines Kristalls durch die skalare Variable $L$ beschrieben wird. Die Verteilungsdichtefunktionen $f_{p}(L, t)$ und $f_{c}(L, t)$ sind die sog. Kristallgrößenverteilungen (KGVen), $f_{p \text {,seed }}(L)$ ist die KGV der Saatkristalle und $G_{p}$ und $B_{p}$ (bzw. $G_{c}$ und $B_{c}$ ) sind die Wachstums- und Nukleationsraten des bevorzugten Enantiomers (bzw. des Gegenenantiomers). Man beachte, dass die Wachstums- und Nukleationsraten nicht explizit von der Zeit $t$ abhängen, sondern Funktionen der Temperatur $T$ des Flüssig-Feststoff-Gemisches, der die Flüssigphase beschreibenden Zustandsgrößen $m_{l, p}$ und $m_{l, c}$ (die absoluten gelösten Massen) sowie (im Fall der Nukleationsraten) integraler Größen der KGVen in dem jeweiligen Kristallisator sind. Dies wird im Folgenden manchmal durch (.) ausgedrückt.

Aus (1) kann ein sog. Momentenmodell abgeleitet werden (siehe z. B. [21]):

$$
\begin{aligned}
\dot{\mu}_{i p} & =i G_{p}(\cdot) \mu_{(i-1) p}, i=1 \ldots 3, \quad \dot{\mu}_{0 p}=B_{p}(\cdot) \\
\dot{\mu}_{i c} & =i G_{c}(\cdot) \mu_{(i-1) c}, i=1 \ldots 3, \quad \dot{\mu}_{0 c}=B_{c}(\cdot)
\end{aligned}
$$

mit den Anfangsbedingungen

$$
\begin{aligned}
& \mu_{i p}(0)=\mu_{i p \text {,seed }}=\int_{0}^{\infty} L^{i} f_{p, \text { seed }}(L) d L, i=0 \ldots 3, \\
& \mu_{i c}(0)=0, \quad i=0 \ldots 3,
\end{aligned}
$$

wobei die Momente definiert sind als

$$
\begin{array}{ll}
\mu_{i p}(t)=\int_{0}^{\infty} L^{i} f_{p}(L, t) d L, & i=0 \ldots 3, \\
\mu_{i c}(t)=\int_{0}^{\infty} L^{i} f_{c}(L, t) d L, & i=0 \ldots 3 .
\end{array}
$$

Das Populationsbilanzmodell (1) bzw. das Momentenmodell (2) wird durch DGLen für die Dynamik der Flüssigphase zum Gesamtmodell vervollständigt. Im asymmetrischen Fall würden diese eine Wechselwirkung der Vorgänge in den beiden Kristallisatoren einschließen. Bei symmetrischen Verhältnissen gilt jedoch [16]

$$
\begin{aligned}
& \dot{m}_{l, p}=-3 \rho_{s} k_{v} G_{p}(\cdot) \mu_{2 p}+\dot{m}_{W, \mathrm{ex}} \frac{m_{l, c}-m_{l, p}}{m_{W}}, \\
& \dot{m}_{l, c}=-3 \rho_{s} k_{v} G_{c}(\cdot) \mu_{2 c}+\dot{m}_{W, \mathrm{ex}} \frac{m_{l, p}-m_{l, c}}{m_{W}},
\end{aligned}
$$

und das Modell der Vorgänge in einem Kristallisator ist in sich geschlossen. Zwecks Übersichtlichkeit der Darstellung wird ein (in beiden Richtungen gleich großer) konstanter Massenstrom $\dot{m}_{W \text {,ex }}$ des Lösungsmittels (Masse $m_{W}$ ) angenommen. Die folgenden Ergebnisse könnten jedoch leicht für den realistischeren Fall eines konstanten 
Volumenstroms angepasst werden, bei dem $\dot{m}_{W \text {,ex }}$ entsprechend $m_{l, p}$ und $m_{l, c}$ und der u.A. temperaturabhängigen Dichte der Lösung geringfügig variiert.

Bei gegebenem Stellgrößenverlauf $T(t)$ lassen sich die zeitlichen Verläufe von $\mu_{0 p}, \ldots, \mu_{3 p}, \mu_{0 c}, \ldots, \mu_{3 c}, m_{l, p}$ und $m_{l, c}$ durch (2), (4) ohne Einbeziehung höherer Momente berechnen; ein in sich geschlossenes Modell in Form eines Systems gewöhnlicher DGLen erster Ordnung $(n=10$ Zustandsgrößen) liegt vor. Hier ist kein konkreter Ausgang definiert. Beim Verfahren zur Berechnung einer Steuerung in Abschnitt 3 kann $B_{c} / G_{c}$ als Ausgangsgröße des Modells (2), (4) angesehen werden und soll (in skalierter Zeit) einem aus einer gewünschten KGV und der PBG (1b) resultierenden Profil folgen.

\subsection{Wachstums- und Nukleationskinetiken}

Im Folgenden wird ein quantitatives Modell der Kristallisation von L-/D-Threonin aus wässriger Lösung vorgestellt, anhand dessen die entwickelten Verfahren erprobt werden sollen. Dabei handelt es sich um das schon in $[15,16]$ verwendete Modell, welches der Arbeit unserer Kooperationspartner der PCF-Gruppe (A. Seidel-Morgenstern) am MPI für Dynamik komplexer technischer Systeme (z. B. [8]) entstammt. Die (durch umfangreiche Messreihen identifizierten) Parameter und die Anfangsbedingungen finden sich in Tabelle 1.

Bei der bevorzugten Kristallisation wird die Übersättigung für jede Enantiomersorte separat definiert und hängt u.A. von den Massenanteilen $w_{l, p}$ und $w_{l, c}$ ab:

$$
S_{\varepsilon}=\frac{w_{l, \varepsilon}}{w_{e q, \varepsilon}}, \quad w_{l, \varepsilon}=\frac{m_{l, \varepsilon}}{m_{l, p}+m_{l, c}+m_{W}},
$$

wobei der Index $\mathcal{E} \in\{p, c\}$ für die entsprechende Enantiomersorte $p$ bzw. $c$ steht. Die Gleichgewichts-Massenanteile $w_{e q, \mathcal{E}}, \mathcal{E} \in\{p, c\}$, werden von der Temperatur des Flüssig-Feststoff-Gemisches über die (empirisch ermittelte) Löslichkeitskorrelationen bestimmt:

$$
w_{e q, \mathcal{E}}=a_{0}+a_{1} \theta, \quad \theta=(T-273.15 \mathrm{~K}){ }^{\circ} \mathrm{C} .
$$

\begin{tabular}{|c|c|c|c|}
\hline Variable & Symbol & Wert & Einheit \\
\hline Dichte der Kristalle & $\rho_{s}$ & $1.25 \times 10^{3}$ & {$\left[\mathrm{~kg} \mathrm{~m}^{-3}\right]$} \\
\hline Volumen-Formfaktor & $k_{v}$ & 0.1222 & {$[-]$} \\
\hline \multirow[t]{3}{*}{ Konstanten für Dichte d. Lösung } & $K_{1}$ & $1.00023 \times 10^{-3}$ & {$\left[\mathrm{~kg}^{-1} \mathrm{~m}^{3}\right]$} \\
\hline & $K_{2}$ & $4.68088 \times 10^{-9}$ & {$\left[\mathrm{~kg}^{-1} \mathrm{~m}^{3} \circ \mathrm{C}^{-2}\right]$} \\
\hline & $K_{3}$ & $0.3652 \times 10^{3}$ & {$\left[\mathrm{~kg} \mathrm{~m}^{-3}\right]$} \\
\hline \multirow[t]{2}{*}{ Löslichkeitskonstanten } & $a_{0}$ & $-3.4505 \times 10^{-2}$ & {$[-]$} \\
\hline & $a_{1}$ & $3.983 \times 10^{-3}$ & {$\left[{ }^{\circ} \mathrm{C}^{-1}\right]$} \\
\hline Universelle Gaskonstante & $R_{g}$ & 8.314472 & {$\left[\mathrm{~J} \mathrm{~K}^{-1} \mathrm{~mol}^{-1}\right]$} \\
\hline Rührergeschwindigkeit & $\omega$ & $2 \pi \times 250$ & {$\left[\min ^{-1}\right]$} \\
\hline \multicolumn{4}{|l|}{ Wachstum } \\
\hline Wachstumskoeffizient & $k_{g 01}$ & $2.7493 \times 10^{7}$ & {$\left[\mathrm{~m} \min ^{-1} \min ^{n_{g}}\right]$} \\
\hline Expon. für Rührergeschwindigkeit & $n_{g}$ & 0.4573 & {$[-]$} \\
\hline Aktivierungsenergie & $E_{A g}$ & $75.549 \times 10^{3}$ & {$\left[\mathrm{~J} \mathrm{~mol}^{-1}\right]$} \\
\hline Wachstumsexponent & $g$ & 1.2269 & {$[-]$} \\
\hline \multicolumn{4}{|l|}{ Primärnukleation } \\
\hline Temperaturabhängigkeit & $K_{T, \text { visc }}$ & 1874.4 & {$[\mathrm{~K}]$} \\
\hline Abhängigkeit v. d. Massenanteilen & $K_{w, \mathrm{visc}}$ & 0.29 & {$[-]$} \\
\hline Exponentielle Abhängigkeit & $k_{\text {prim,log }}$ & $4.304 \times 10^{-3}$ & {$[-]$} \\
\hline Heterogene Primärnukleation & $A_{1}$ & $6.5608 \times 10^{-4}$ & {$\left[\mathrm{~m}^{-2}\right]$} \\
\hline \multicolumn{4}{|l|}{ Sekundärnukleation } \\
\hline Sekundärnukleationskoeffizient & $k_{b, \sec 01}$ & $3.2635 \times 10^{30}$ & {$\left[\min ^{-1} \mathrm{~m}^{-3 n_{\mu_{3}}}\right]$} \\
\hline Expon. für Rührergeschwindigkeit & $n_{\mathrm{sec}, b}$ & $4.9295 \times 10^{3}$ & {$\left[\min ^{-1}\right]$} \\
\hline Aktivierungsenergie & $E_{A b, \text { sec }}$ & $63.83 \times 10^{3}$ & {$\left[\mathrm{~J} \mathrm{~mol}^{-1}\right]$} \\
\hline Sekundärnukleationsexponent & $b_{\mathrm{sec}}$ & 16.2477 & {$[-]$} \\
\hline Exponent für drittes Moment & $n_{\mu_{3}}$ & 0.65317 & {$[-]$} \\
\hline \multicolumn{4}{|l|}{ Anfangsbedingungen } \\
\hline Masse d. Lösungsmittels (Wasser) & $m_{W}$ & 0.35919 & {$[\mathrm{~kg}]$} \\
\hline Gelöste Massen $m_{l, p}(0)=m_{l, c}(0)$ & $m_{l}(0)$ & 0.045405 & {$[\mathrm{~kg}]$} \\
\hline \multirow[t]{2}{*}{ Momente d. KGV der Saatkristalle } & $\mu_{0 p}(0)$ & $1.4412 \times 10^{4}$ & {$[-]$} \\
\hline & $\mu_{1 p}(0)$ & 7.8331 & {$[\mathrm{~m}]$} \\
\hline Momente d. Gegenenantiomer-KGV & $\mu_{i c}(0)$ & 0 & {$\left[\mathrm{~m}^{i}\right]$} \\
\hline
\end{tabular}

Tabelle 1: In dem Rechenbeispiel verwendete Parameter und Anfangsbedingungen. 
Die Wachstumsrate der entsprechenden Enantiomersorte $\mathcal{E} \in\{p, c\}$ berechnet sich über

$$
G_{\mathcal{E}}(\cdot)=k_{g 01} \cdot \omega^{n_{g}} \cdot \exp \left(-\frac{E_{A g}}{R_{g} T}\right)\left(S_{\mathcal{E}}-1\right)^{g} .
$$

Die Nukleationsrate jeder Enantiomersorte setzt sich zusammen aus Primärnukleation $B_{\text {prim, } \mathcal{E}}$ und Sekundärnukleation $B_{\mathrm{sec}, \mathcal{E}}$ :

$$
B_{\mathcal{E}}=B_{\text {prim }, \mathcal{E}}+B_{\text {sec }, \mathcal{E}}, \quad \mathcal{E} \in\{p, c\},
$$

mit

$$
\begin{aligned}
& B_{\text {prim }, \mathcal{E}}=k_{b, \text { prim }} \cdot T \cdot \exp \left(-\frac{K_{T, \text { visc }}}{T}\right) \\
& \cdot \exp \left(-\frac{w_{l, p}+w_{l, c}}{K_{w, \text { visc }}}\right) \sqrt{\ln \left(\frac{\rho_{s}}{C_{e q, \mathcal{E}}}\right)} \cdot\left(S_{\mathcal{E}} C_{e q, \mathcal{E}}\right)^{7 / 3} \\
& \cdot \exp \left(-k_{\text {prim,log }} \frac{\left(\ln \left(\rho_{s} / C_{e q, \mathcal{E}}\right)\right)^{3}}{\left(\ln \left(S_{\mathcal{E}}\right)\right)^{2}}\right) \cdot\left(1+A_{1} \mu_{2} \bar{\varepsilon}\right), \\
& C_{e q, \mathcal{E}}=\rho_{\text {sol }} \cdot w_{e q, \mathcal{E}}=\left(\rho_{W}+K_{3}\left(w_{l, p}+w_{l, c}\right)\right) \cdot w_{e q, \mathcal{E}} \\
& \rho_{W}=1 /\left(K_{1}+K_{2} \theta^{2}\right), \quad \theta=(T-273.15 \mathrm{~K}) \frac{{ }^{\circ} \mathrm{C}}{\mathrm{K}},
\end{aligned}
$$

und

$$
\begin{array}{r}
B_{\mathrm{sec}, \mathcal{E}}=k_{b, \mathrm{sec} 01} \cdot \exp \left(-\frac{n_{\mathrm{sec}, b}}{\omega}\right) \exp \left(-\frac{E_{A b, \mathrm{sec}}}{R_{g} T}\right) \\
\cdot\left(S_{\mathcal{E}}-1\right)^{b_{\mathrm{sec}}}\left(\mu_{3 \mathcal{E}}\right)^{n_{\mu 3}} .
\end{array}
$$

In (8b) kennzeichnet $\bar{\varepsilon}$ die jeweils andere Enantiomersorte. Der empirische Term $\left(1+A_{1} \mu_{2} \bar{\varepsilon}\right)$ beschreibt den Effekt der sog. heterogenen Primärnukleation.

\section{Modellinversion zur Realisierung gewünschter KGVen}

Die KGV des kristallinen Feststoffs steht oft im Fokus der Steuerung und Regelung von Kristallisationsprozessen, beispielsweise zur Begünstigung der Aufnahme eines Medikaments - siehe z. B. die einleitende Literaturübersicht in [16]. In [15] wurde für die bevorzugte Kristallisation (Batch) durch (dynamische) Inversion des Modells eine Temperatursteuerung (d.h. der erforderliche zeitliche Verlauf der Stellgröße Temperatur) zur Realisierung einer gewünschten End-KGV einer Enantiomersorte berechnet. In [16] wurde eine Erweiterung vorgeschlagen, bei der durch
Inversion eines Modells der gekoppelten Kristallisatoren in jedem Kristallisator jeweils die End-KGV einer der beiden Enantiomersorten realisiert werden konnte. Im vorliegenden Aufsatz kann eine solche Inversion des gekoppelten Modells aufgrund der getroffenen Symmetrieannahmen auf deutlich einfachere Weise erfolgen. Insbesondere kann das gesamte Inversionsverfahren in einer geeignet transformierten Zeit durchgeführt werden. Um die Symmetrieannahmen zu wahren, muss die Spezifikation der gewünschten KGVen ebenfalls symmetrisch sein. Entweder wird für jeden Kristallisator jeweils die KGV des bevorzugten Enantiomers (also $E_{1}$ in Kristallisator $A$ und $E_{2}$ in Kristallisator $B$ ), oder jeweils die KGV des Gegenenantiomers (also $E_{2}$ in Kristallisator $A$ und $E_{1}$ in Kristallisator $B$ ) spezifiziert. In [16] wurde erklärt, dass und warum beide Fälle sinnvoll sein können.

\subsection{Inversion im symmetrischen Fall}

Für die Lösung der Inversionsaufgabe zur Realisierung einer vorgegebenen End-KGV im Fall der EinstoffKristallisation wurde in $[27,28]$ zunächst eine Zeittransformation entsprechend der Wachstumsrate der Kristalle angewendet. Diese Transformation verwandelt die PBG in eine einfache Transportgleichung. Um dies im hier betrachteten Kontext für eine der beiden Enantiomersorten $\mathcal{E} \in\{p, c\}$ zu erreichen, muss die Zeit mit der entsprechenden Wachstumsrate $G_{\mathcal{E}}(\cdot)$ skaliert werden. Die resultierende Zeitvariable wird mit $\tau_{\mathcal{E}}$ bezeichnet:

$$
d \tau_{\mathcal{E}}=G_{\mathcal{E}}(\cdot) d t, \quad \tau_{\mathcal{\varepsilon}}(t=0)=0 .
$$

Da die Wachstumsraten des verwendeten Modells nicht explizit von den KGVen abhängen, ergibt die Anwendung dieser Zeitskalierung auf (1a) (für $\mathcal{E}=p$ ) bzw. (1b) (für $\varepsilon=c)$ :

$$
\frac{\partial f_{\mathcal{E}}\left(L, \tau_{\mathcal{E}}\right)}{\partial \tau_{\mathcal{E}}}=-\frac{\partial f_{\mathcal{E}}\left(L, \tau_{\mathcal{E}}\right)}{\partial L},
$$

mit den Rand- und Anfangsbedingungen

$$
\begin{aligned}
f_{\mathcal{E}}\left(0, \tau_{\mathcal{E}}\right) & =\frac{B_{\mathcal{E}}\left(\tau_{\mathcal{E}}\right)}{G_{\mathcal{E}}\left(\tau_{\mathcal{E}}\right)}, \\
f_{\mathcal{E}}(L, 0) & = \begin{cases}f_{\mathcal{E}, \text { seed }}(L), & \text { für } \mathcal{E}=p, \\
0, & \text { für } \mathcal{E}=c .\end{cases}
\end{aligned}
$$

Die charakteristischen Kurven (Kurven, auf denen $f_{\mathcal{E}}$ konstant ist) der PBG (10a) sind gerade Linien, siehe Abbildung 2 (b), während dies für (1a) bzw. (1b) i. A. nicht der Fall ist. Auf diese Weise folgt bei Vorgabe des nukleierten Teils $f_{\mathcal{E}}\left(L, \tau_{\mathcal{E}, f}\right)=f_{\mathcal{E}, n}\left(L, \tau_{\mathcal{E}, f}\right), L \in\left[0, L_{\max }=\tau_{\mathcal{E}, f}\right]$, der 


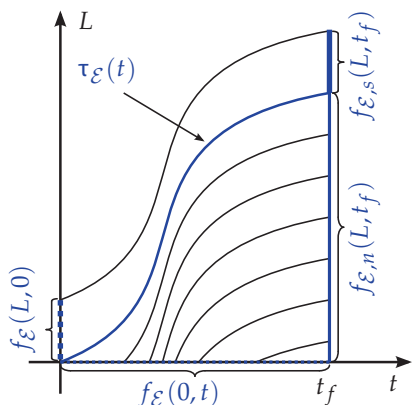

a)

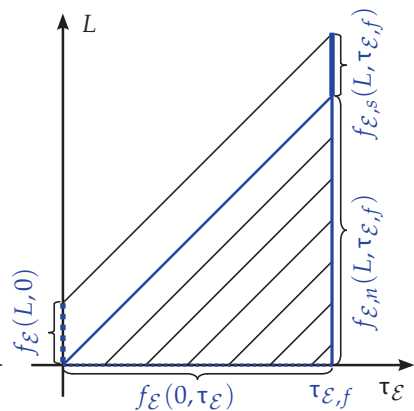

b)
Abbildung 2: Charakteristische Kurven der Populationsbilanzgleichung für eine Enantiomersorte $\mathcal{E} \in\{p, c\}$ (a) ohne Zeitskalierung, (b) der Fall mit Zeitskalierung (9).

End-KGV einer Enantiomersorte unmittelbar der Verlauf der entsprechenden Randbedingung in der transformierten Zeit $\tau_{\varepsilon}$ :

$$
\frac{B_{\varepsilon}\left(\tau_{\varepsilon}\right)}{G_{\varepsilon}\left(\tau_{\varepsilon}\right)}=f_{\mathcal{\varepsilon}}\left(\tau_{\varepsilon, f}-\tau_{\varepsilon}, \tau_{\varepsilon, f}\right), \quad \tau_{\varepsilon} \in\left[0, \tau_{\varepsilon, f}\right] .
$$

Der andere Teil $f_{\mathcal{E}}\left(L, \tau_{\mathcal{\varepsilon}, f}\right)=f_{\mathcal{E}, s}\left(L, \tau_{\varepsilon, f}\right), L>\tau_{\varepsilon, f}$, der End-KGV ergibt sich hingegen auf eindeutige und einfache Weise aus der zugehörigen Anfangsbedingung:

$$
f_{\mathcal{E}}\left(L, \tau_{\mathcal{E}, f}\right)=\left\{\begin{array}{lll}
f_{\mathcal{E}, \text { seed }}\left(L-\tau_{\mathcal{E}, f}\right), & L>\tau_{\mathcal{E}, f}, & \mathcal{E}=p, \\
0, & L>\tau_{\mathcal{E}, f}, & \mathcal{E}=c .
\end{array}\right.
$$

Dieser KGV-Teil kann somit nicht frei vorgegeben werden. Allerdings lässt sich die gewünschte Längenzunahme der Saatkristalle bei der Vorgabe von $f_{p, n}$ durch $L_{\max }=\tau_{p, f}$ implizit berücksichtigen.

Die Gleichung (11) kann als erster Schritt des Inversionsverfahrens zur Realisierung der KGV-Vorgabe $f_{\mathcal{E}, n}\left(L, \tau_{\mathcal{E}, f}\right) \stackrel{!}{=} f_{\mathcal{E}, \mathrm{des}}(L), L \in\left[0, L_{\text {max }}\right]$, angesehen werden. Im zweiten Schritt muss ein geeignetes Stellgrößenprofil $T(t)$ zur Realisierung des Profils $B_{\varepsilon}\left(\tau_{\varepsilon}\right) / G_{\varepsilon}\left(\tau_{\varepsilon}\right)$ ermittelt werden. Dies bedarf - wie in $[15,16]$ demonstriert - der dynamischen Inversion des Modells (2), (4) und wird im Folgenden für den Spezialfall symmetrischer Verhältnisse in abgekürzter Form dargestellt.

Mit Blick auf die folgenden Abschnitte wird nun von einer Vorgabe $f_{c \text {,des }}(L), L \in\left[0, L_{\text {max }}\right]$ einer Gegenenantiomer-KGV ausgegangen. Somit ist durch (11) ein zu realisierendes Profil in der $\tau_{c}$-Zeit vorgegeben. Es liegt nahe, für die Berechnung des erforderlichen Temperaturprofils auch das Momentenmodell (2) und die Dynamik der Flüs- sigphase (4) in die $\tau_{c}$-Zeit zu transformieren:

$$
\begin{aligned}
& \frac{d \mu_{i p}}{d \tau_{c}}=i \frac{G_{p}(\cdot)}{G_{c}(\cdot)} \mu_{(i-1) p}, i=1 \ldots 3, \frac{d \mu_{0 p}}{d \tau_{c}}=\frac{B_{p}(\cdot)}{G_{c}(\cdot)}, \\
& \frac{d \mu_{i c}}{d \tau_{c}}=i \mu_{(i-1) c}, i=1 \ldots 3, \frac{d \mu_{0 c}}{d \tau_{c}}=\frac{B_{c}(\cdot)}{G_{c}(\cdot)} \\
& \frac{d m_{l, p}}{d \tau_{c}}=-3 \rho_{s} k_{v} \frac{G_{p}(\cdot)}{G_{c}(\cdot)} \mu_{2 p}+\frac{\dot{m}_{W, \mathrm{ex}}}{G_{c}(\cdot)} \frac{m_{l, c}-m_{l, p}}{m_{W}} \\
& \frac{d m_{l, c}}{d \tau_{c}}=-3 \rho_{s} k_{v} \mu_{2 c}+\frac{\dot{m}_{W, \mathrm{ex}}}{G_{c}(\cdot)} \frac{m_{l, p}-m_{l, c}}{m_{W}} .
\end{aligned}
$$

(13), (14) sind gekoppelt mit der algebraischen Gleichung1

$$
\frac{B_{c}(T, \cdot)}{G_{c}(T, \cdot)}=f_{c, \text { des }}\left(\tau_{c, f}-\tau_{c}\right), \quad \tau_{c} \in\left[0, \tau_{c, f}\right] .
$$

Für die in Abschnitt 2.2 eingeführten Wachstums- und Nukleationskinetiken kann leicht überprüft werden, dass die Modell-DGLen innerhalb eines physikalisch sinnvollen Definitionsbereichs $D$ kontinuierlich nach den Zustandsgrößen $\left(\mu_{i p}, i=0 \ldots 3, \mu_{i c}, i=0 \ldots 3, m_{l, p}, m_{l, c}\right)$ sowie der Stellgröße $(T)$ differenzierbar sind. Man beachte, dass $G_{c}(\cdot) \neq 0$ innerhalb $D$ gelten muss. Wenn zusätzlich (15) eindeutig nach $T$ auflösbar und die resultierende Funktion kontinuierlich nach den Zustandsgrößen und $\tau_{c}$ differenzierbar ist, kann das System (13), (14), (15) durch Substitution dieser Funktion in (13), (14) auf ein System gewöhnlicher DGLen (mit expliziter Abhängigkeit von der "Zeit“ $\tau_{c}$ ) zurückgeführt werden. Letzteres kann - für physikalisch mögliche KGV-Vorgaben - unter den getroffenen Annahmen eindeutig gelöst werden (z. B. [17]), wobei die „Zeit“Dauer $\tau_{c, f}=L_{\max }$ von vornherein feststeht. Anschließend müssen die erhaltenen Verläufe (z. B. der Temperaturverlauf $T\left(\tau_{c}\right)$ ) in die $t$-Zeit zurück transformiert werden:

$$
t\left(\tau_{c}\right)=\int_{0}^{\tau_{c}}\left(G_{c}\left(\tau_{c}^{\prime}\right)\right)^{-1} d \tau_{c}^{\prime} .
$$

Daraus folgt auch die „echte“ Dauer des Batchprozesses.

In [27] wurde gezeigt, dass das Momentenmodell eines Einstoff-Kristallisationssystems für größenunabhängige Wachstumsrate orbitell flach, d.h. differenziell flach

1 Formal kann $B_{c}\left(\tau_{c}\right) / G_{c}\left(\tau_{c}\right)$ als Ausgangsgröße des Modells (13), (14) angesehen werden, deren Profil vorgegeben ist. In [15] wird ein sehr ähnliches System (zeitskaliertes Momentenmodell der bevorzugten Kristallisation in einem Gefäß) durch dynamische Erweiterung auf eine Form gebracht, die affin im Eingang ist, und $\mu_{3 p}$ bzw. $\mu_{3 c}$ wird formal als Ausgang definiert. Das nicht flache System wird in eine Normalform transformiert und invertiert, wobei die Nulldynamik und der relative Grad explizit angegeben werden. 
in einer geeigneten transformierten Zeit ist. Für eine Beschreibung dieser sehr nützlichen Systemeigenschaften siehe z. B. [9, 22, 23, 25]. Leider ist das System (13), (14) nicht differenziell flach, so dass sich das Lösen von DGLen bei der dynamischen Inversion nicht vermeiden lässt. Mit den auf dem sog. Regelflächenkriterium (siehe z. B. [25, 26]) basierenden Ergebnissen aus [15] kann für übliche (wie die hier verwendeten) Wachstums- und Nukleationskinetiken sogar gezeigt werden, dass das System (2), (4) nicht orbitell flach ist. Es existiert also keine zulässige Zeitskalierung, die das System in ein flaches System überführt.

Die Auflösbarkeit von (15) nach $T$ sowie die kontinuierliche Differenzierbarkeit dieser aufgelösten Form nach den Zustandsgrößen und $\tau_{c}$ kann mit Hilfe des Satzes über das Auflösen impliziter Funktionen (z. B. [17]) sichergestellt werden, wenn $f_{c \text {,des }}(L)$ kontinuierlich differenzierbar ist und die Ableitung des Quotienten $B_{c}(T, \cdot) / G_{c}(T, \cdot)$ nach $T$ ungleich Null ist. Die letztere Bedingung ist im Fall komplexer Wachstums- und Nukleationskinetiken jedoch nicht selbstverständlich erfüllt. Die Existenz mehrerer Lösungen kann aber z. B. durch eine Einschränkung des Definitionsbereichs (also des zulässigen Temperaturbereichs) garantiert werden.

Es sei angemerkt, dass sich ein entsprechendes dynamisches Inversionsproblem auch bei asymmetrischen Verhältnissen lösen lässt. In diesem Fall können sich die KGVVorgaben für die beiden Kristallisatoren auch unterscheiden. Man hat es dann i. A. mit unterschiedlichen Wachstumsraten der interessierenden Kristallpopulationen $\mathrm{zu}$ tun. Auch werden die Kristallisationsvorgänge in beiden Gefäßen i. A. nicht gleichzeitig beendet sein. Dies erfordert - wie in [16] dargestellt - eine etwas kompliziertere Vorgehensweise als oben beschrieben.

Im Folgenden soll stattdessen untersucht werden, inwiefern sich das Inversionsproblem weiter vereinfacht, wenn zusätzliche idealisierende Annahmen getroffen werden.

\subsection{Modellinversion bei weiteren Idealisierungen}

\section{Idealer Flüssigkeitsaustausch}

Der hypothetische Fall eines unendlich schnellen Flüssigkeitsaustauschs hätte eine perfekte Durchmischung der Lösungen in den Kristallisatoren zur Folge. Beispielsweise wäre dann die an $E_{1}$ gelöste Masse dieselbe in den Kristallisatoren $A$ und $B$. Zusammen mit den vorausgesetzten symmetrischen Verhältnissen impliziert dies für jeden
Kristallisator jeweils $m_{l, p}(t)=m_{l, c}(t)$. Die gelösten Massen lassen sich dann durch Heranziehen einer beide Kristallisatoren umfassenden Massenbilanz berechnen, bilden also keine Zustandsgrößen mehr:

$$
\begin{aligned}
& m_{l}(t):=m_{l, p}(t)=m_{l, c}(t) \\
& =m_{l}(0)-0.5 \rho_{s} k_{v}\left(\mu_{3 p}(t)-\mu_{3 p}(0)+\mu_{3 c}(t)-\mu_{3 c}(0)\right),
\end{aligned}
$$

wobei natürlich $m_{l}(0)=m_{l, p}(0)=m_{l, c}(0)$ gelten muss.

Aus der Symmetrie und der identischen Zusammensetzung der Flüssigphase in den beiden Kristallisatoren folgt zudem dass

$$
G_{p}(\cdot)=G_{c}(\cdot)=: G(\cdot) .
$$

Durch Einsetzen der Massenbilanz (17) in die entsprechenden Modellgleichungen lassen sich die Wachstumsrate $G(\cdot)=G_{p}(\cdot)=G_{c}(\cdot)$ (7) und die Nukleationsraten $B_{p}(\cdot)$ und $B_{c}(\cdot)(8)$ als Funktionen von $T, \mu_{2 p}, \mu_{3 p}, \mu_{2 c}$, $\mu_{3 c}$ schreiben. Nach Einführung der Zeitskalierung

$$
d \tau=G(\cdot) d t, \quad \tau(t=0)=0,
$$

erhält man aus (2) das (geschlossene) Momentenmodell

$$
\begin{aligned}
\frac{d}{d \tau} \mu_{i p} & =i \mu_{(i-1) p}, \quad i=1 \ldots 3, \\
\frac{d}{d \tau} \mu_{0 p} & =\frac{B_{p}\left(T, \mu_{2 c}, \mu_{3 p}, \mu_{3 c}\right)}{G\left(T, \mu_{3 p}, \mu_{3 c}\right)}, \\
\frac{d}{d \tau} \mu_{i c} & =i \mu_{(i-1) c}, \quad i=1 \ldots 3, \\
\frac{d}{d \tau} \mu_{0 c} & =\frac{B_{c}\left(T, \mu_{2 p}, \mu_{3 p}, \mu_{3 c}\right)}{G\left(T, \mu_{3 p}, \mu_{3 c}\right)} .
\end{aligned}
$$

Trotz der identischen Wachstumsraten kann gezeigt werden, dass dieses System weder differenziell noch orbitell flach ist [16]. Die folgende zusätzliche Idealisierung verwandelt (20) jedoch in ein differenziell flaches System.

\section{Vernachlässigung der Nukleation der bevorzugten Enantiomere}

In $[15,16]$ wird argumentiert, dass nicht nur die (intuitiv einleuchtende) Vernachlässigung der Nukleation des Gegenenantiomers, sondern stattdessen auch die Vernachlässigung der Nukleation des gewünschten Enantiomers gerechtfertigt und sinnvoll sein kann. Letzteres ist beispielsweise gegeben, wenn - wie in der Praxis häufig anzutreffen - die angestrebte KGV des gewünschten Enantiomers fast ausschließlich durch Wachsen von Saatkristallen zustande kommen soll. Dies kann bei gegebener Qualität und Menge der Saatkristalle offensichtlich nur erreicht 
werden, wenn die zugehörige Nukleationsrate durch geeignete Stelleingriffe hinreichend klein gehalten wird. $\mathrm{Ob}$ diese Idealisierung in Bezug auf die Anforderungen an die KGV der bevorzugten Enantiomere sowie deren modellinterne Rückwirkung tatsächlich gerechtfertigt ist, muss gegebenenfalls a-posteriori überprüft werden.

Für $B_{p}(\tau) \equiv 0$ wird die KGV der bevorzugten Enantiomere nur durch das Wachstum der Saatkristalle bestimmt. Für das 2. und 3. Moment bedeutet dies [15]

$$
\begin{aligned}
\mu_{2 p}(\tau)=\mu_{2 p, s}(\tau)= & \mu_{0 p, \text { seed }} \tau^{2} \\
& +2 \mu_{1 p, \text { seed }} \tau+\mu_{2 p \text {,seed }} \\
\mu_{3 p}(\tau)=\mu_{3 p, s}(\tau)= & \mu_{0 p \text {,seed }} \tau^{3}+3 \mu_{1 p \text {,seed }} \tau^{2} \\
& +3 \mu_{2 p, \text { seed }} \tau+\mu_{3 p \text {,seed }} .
\end{aligned}
$$

Dadurch werden (20a), (20b) überflüssig, und durch Einsetzen von (21) in (20c), (20d) entsteht ein abgeschlossenes Momentenmodell für die Kristallisation des Gegenenantiomers:

$$
\begin{aligned}
\frac{d}{d \tau} \mu_{i c} & =i \mu_{(i-1) c}, \quad i=1 \ldots 3, \\
\frac{d}{d \tau} \mu_{0 c} & =\frac{B_{c}\left(T, \tau, \mu_{3 c}\right)}{G\left(T, \tau, \mu_{3 c}\right)} .
\end{aligned}
$$

Das Modell (22) hat im Grunde die Form eines (zeitskalierten) Modells der Einstoff-Kristallisation (gemäß [27, 28]). Einziger struktureller Unterschied ist das explizite Erscheinen der $\tau$-Zeit in den Termen für $B_{c}(\cdot)$ und $G(\cdot)$. Wenn sich die rechte Seite von (22b) eindeutig nach der Temperatur $T$ auflösen lässt, ist (22) ein flaches System: Die Momentanwerte aller Zustandsgrößen und der Stellgröße $T(\tau)$ lassen sich bei Kenntnis des flachen Ausgangs $\mu_{3 c}(\tau)$ und einer endlichen $\mathrm{Zahl}$ von dessen Ableitungen algebraisch berechnen. Die Berechnungsvorschrift für $T$ beinhaltet jedoch eine explizite Abhängigkeit von der skalierten Zeit $\tau$. Derartige Beispiele flacher Systeme finden sich auch in $[2,25]$.

\section{Minimierung der Batchdauer}

Im Folgenden soll ein Problem der optimalen Steuerung gelöst werden. Die Laufzeit des Batchprozesses soll unter der Nebenbedingung minimiert werden, dass eine vorgegebene Menge $\mu_{3 p, s, \text { des }}$ des bevorzugten Enantiomers aus gewachsenen Saatkristallen entsteht und die Verunreinigung $\operatorname{imp}_{f}:=\mu_{3 c, f} /\left(\mu_{3 p, f}+\mu_{3 c, f}\right)$ durch das Gegenenantiomer eine zulässige Obergrenze $\mathrm{imp}_{\text {des }}$ nicht überschreitet:

$$
\min _{T(t)} t_{f} \text { so dass } \begin{aligned}
\mu_{3 p, f, s} & =\mu_{3 p, s, \mathrm{des}} \\
\operatorname{imp}_{f} & \leq \mathrm{imp}_{\mathrm{des}} .
\end{aligned}
$$

\subsection{Lösung auf Basis des idealisierten Modells}

Hier wird eine Methode vorgestellt, um das dynamische Optimierungsproblem (23) für das nach Abschnitt 3.2 idealisierte Modell (idealer Flüssigkeitsaustausch und Vernachlässigung der Nukleation der bevorzugten Enantiomere) zu lösen. Dieses Modell ist, wie oben angedeutet, orbitell flach. Zunächst kann $\tau_{f}$ durch Lösen von (21b) für $\tau=$ $\tau_{f}$ und $\mu_{3 p, s}\left(\tau_{f}\right)=\mu_{3 p, s, \text { des }}$ bestimmt werden. Die Lösung wird im Folgenden mit $\tau_{\text {des }}$ bezeichnet. Durch die getroffene idealisierende Annahme gilt $\operatorname{imp}_{f}=\mu_{3 c, f} /\left(\mu_{3 p, f, s}+\right.$ $\left.\mu_{3 c, f}\right)$. Aus der Ungleichheitsnebenbedingung in (23) folgt daher

$$
\mu_{3 c, f} \leq \underbrace{\mu_{3 p, f, s}}_{\mu_{3 p, s, \mathrm{des}}} \frac{\mathrm{imp}_{\mathrm{des}}}{1-\mathrm{imp}_{\mathrm{des}}}=: \mu_{3 c, \mathrm{des}} .
$$

Als äquivalentes Optimierungsproblem in der $\tau$-Zeit erhält man deswegen:

$$
\begin{aligned}
\min _{T(\tau)} t_{f} \text { so dass } & =\tau_{\text {des }} \\
\mu_{3 c}\left(\tau_{f}\right) & \leq \mu_{3 c, \text { des }} .
\end{aligned}
$$

Wie in [12] gezeigt, lassen sich dynamische Optimierungsprobleme für (orbitell) flache Systeme durch Parametrierung des zeitlichen Verlaufes des flachen Ausgangs auf Parameteroptimierungsprobleme zurückführen und daher effizient lösen. Hier wird stattdessen der Ansatz aus [27] aufgegriffen und alternativ der durch Nukleation entstandene Anteil der End-KGV parametriert. Wegen der getroffenen idealisierenden Annahmen bedeutet dies eine Parametrierung von $f_{c}\left(L, \tau_{f}\right)$. Im Folgenden wird eine Interpolation von Stützpunkten $\left(l_{i}, z_{i}\right), i=1, \ldots, K$, durch kubische Splines verwendet. Hierbei gilt $l_{i}=L_{\max }(i-$ 1) $/(K-1)$ mit (siehe Abschnitt 3.1) $L_{\max }=\tau_{f}$. Der positiv reellwertige Vektor $\mathbf{z}=\left(z_{1}, \ldots, z_{K}\right)$ enthält die freien $\mathrm{Pa}$ rameter, d.h.

$$
f_{c}\left(L, \tau_{f}\right)=f_{c}^{\mathrm{z}}(L) .
$$

Somit ist $f_{c}^{\mathrm{z}}(L)$ kontinuierlich nach $L$ differenzierbar. Mit $\tau_{f}=\tau_{\text {des }}$ ist die Gleichheitsnebenbedingung in (25) automatisch erfüllt. Das resultierende Parameteroptimierungsproblem lautet:

$$
\min _{\mathbf{z}} . t_{f}=\int_{0}^{\tau_{f}} \frac{1}{G\left(T(\tau), \tau, \mu_{3 c}(\tau)\right)} d \tau,
$$

so dass

$$
\mu_{3 c}\left(\tau_{f}\right)=\int_{0}^{\tau_{f}} L^{3} f_{c}^{\mathbf{z}}(L) d L \leq \mu_{3 c, \text { des }} .
$$


Hierbei erhält man $T(\tau)$ durch Lösen der Gleichung

$$
\frac{B_{c}\left(T, \tau, \mu_{3 c}(\tau)\right)}{G\left(T, \tau, \mu_{3 c}(\tau)\right)}=f_{c}^{\mathrm{z}}\left(\tau_{f}-\tau\right),
$$

und $\mu_{3 c}(\tau)$ durch viermalige Integration von $f_{c}^{\mathrm{z}}\left(\tau_{f}-\tau\right)$.

Für die Berechnung der Kostenfunktion bei gegebenem Parametervektor $\mathbf{z}$ müssen also nur Integrale berechnet, und keine Differenzialgleichungen gelöst werden. Erwartungsgemäß kann die Lösung jedoch nicht in geschlossener Form ausgedrückt werden. In der Implementierung werden Integrale jeweils numerisch durch Trapezintegration berechnet, und (28) wird mit einem Bisektionsverfahren (25 Iterationen) gelöst. Für die Speicherung der zeitlichen Verläufe ( $\tau$-Zeit) werden jeweils 1000 Stützpunkte verwendet. Der Parametervektor $\mathbf{z}$ hat die Dimension 15. Für die Optimierung wird ein Gradientenverfahren verwendet, wobei der Gradient jeweils durch finite Differenzen (Perturbation der Elemente von $\mathbf{z}$ ) approximiert wird. Als Startwert $\mathbf{z}^{(0)}$ wird ein Vektor mit identischen Einträgen verwendet. Das Gradientenverfahren wird nach einer (durch Inspektion der Zielfunktionsentwicklung als adäquat erachteten) festen Zahl von 30 Iterationen beendet.

Für das Rechenbeispiel wird die Optimierung für eine Reihe von Vorgaben bzgl. Verunreinigung und Produktmenge wiederholt:

$$
\begin{aligned}
& \operatorname{imp}_{\text {des }} \in\left\{10^{-4}, 10^{-5}, 10^{-6}\right\}, \\
& \mu_{3 p, s, \text { des }} \in\left\{100 \mathrm{~cm}^{3}, 200 \mathrm{~cm}^{3}, 300 \mathrm{~cm}^{3}\right\} .
\end{aligned}
$$

Die Vorgabe äußerst geringer Verunreinigungen kann je nach Anwendung (z. B. in der Pharmaindustrie) erforderlich sein. Ausgewählte Ergebnisse für $\mu_{3 p, s, \text { des }}=200 \mathrm{~cm}^{3}$

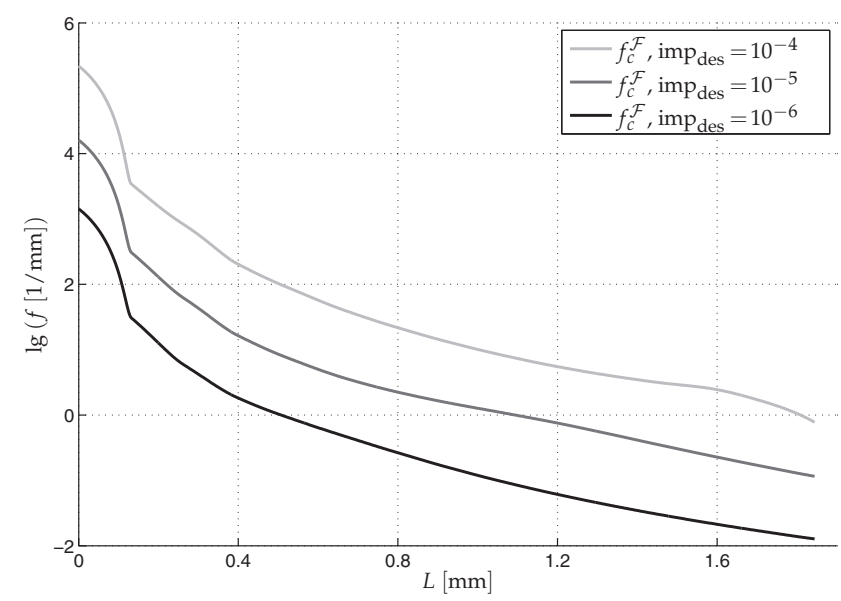

Abbildung 3: KGV-Profile (logarithmisch) der Gegenenantiomere resultierend aus dem Verfahren in Abschnitt $4.1(\mathcal{F})$; $\mu_{3 p, s, \text { des }}=200 \mathrm{~cm}^{3}$.

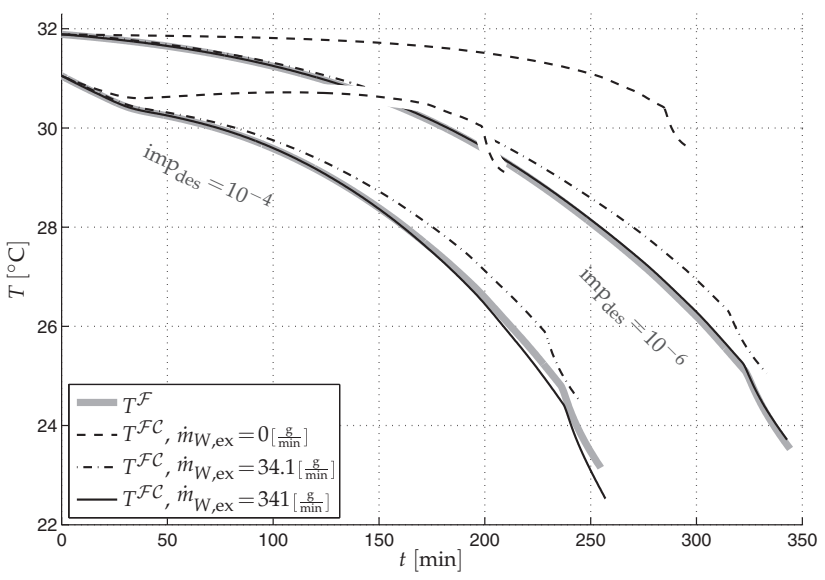

Abbildung 4: Temperaturprofile resultierend aus den Verfahren in Abschnitt $4.1(\mathcal{F})$ und $4.2 \mathrm{~b})(\mathcal{F} \mathcal{C}) ; \mu_{3 p, s, \text { des }}=200 \mathrm{~cm}^{3}$.

sind in den Abbildungen 3 (optimale End-KGV $f_{c}^{* \mathrm{z}}(L)=$ : $f_{c}^{\mathcal{F}}(L)$ des Gegenenantiomers) und 4 (Temperaturverlauf $\left.T^{\mathcal{F}}(t)\right)$ dargestellt. Auffällig ist, dass die Temperatur weder besonders steil abfällt noch besonders kleine Werte annimmt, obwohl entsprechende Beschränkungen nicht explizit berücksichtigt wurden.

Die Berechnungszeit für die Optimierung lag jeweils bei etwa 54 Sekunden. Alle Ergebnisse sind auf demselben Rechner mit MATLAB ${ }^{\circledR}$ entstanden.

\subsection{Approximative Lösungen für das nicht idealisierte Modell}

Dem Optimierungsverfahren nach Abschnitt 4.1 liegen (neben der Symmetrieannahme) die Idealisierungen eines idealen Flüssigkeitsaustauschs sowie der Beschränkung der Kristallisation der bevorzugten Enantiomere auf das Wachstum der Saatkristalle zu Grunde. Bei Anwendung der so erhaltenen Temperaturprofile auf das nicht idealisierte Modell mit endlicher Flüssigkeitsaustauschrate $\dot{m}_{W \text {,ex }}$ und Nukleation der bevorzugten Enantiomere sind entsprechende Fehler $\mathrm{zu}$ erwarten, die quantitativ vom durch die Idealisierung verursachten Modellfehler abhängen werden.

\section{a) Anwendung der idealisierungsbasierten Lösung}

Zunächst soll die Größe der Fehler für verschiedene Werte der Flüssigkeitsaustauschrate $\dot{m}_{W \text {,ex }}$,

$$
\dot{m}_{W, \text { ex }} \in\left\{0 \frac{\mathrm{g}}{\min }, 10.2 \frac{\mathrm{g}}{\min }, 34.1 \frac{\mathrm{g}}{\min }, 102 \frac{\mathrm{g}}{\min }, 341 \frac{\mathrm{g}}{\min }\right\},
$$




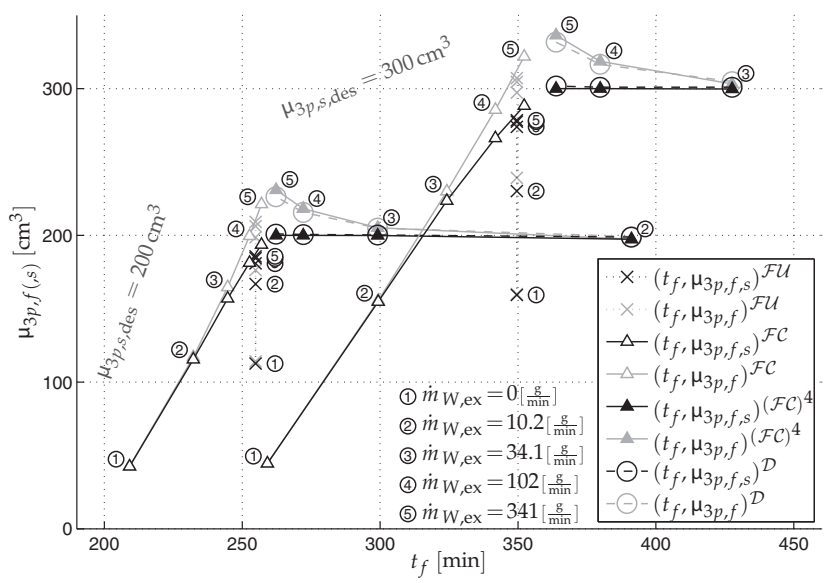

Abbildung 5: Wertepaare $\left(t_{f}, \mu_{3 p, f, s}\right)$ und $\left(t_{f}, \mu_{3 p, f}\right)$ bei Anwendung der Vorgehensweisen aus Abschnitt 4.2 a) (FU), 4.2 b) (FC), $4.2 \mathrm{c}$ ) $\left((\mathcal{F C})^{4}\right)$ sowie $4.3(\mathcal{D}) ;$ imp des $_{\text {des }}=10^{-4}$.

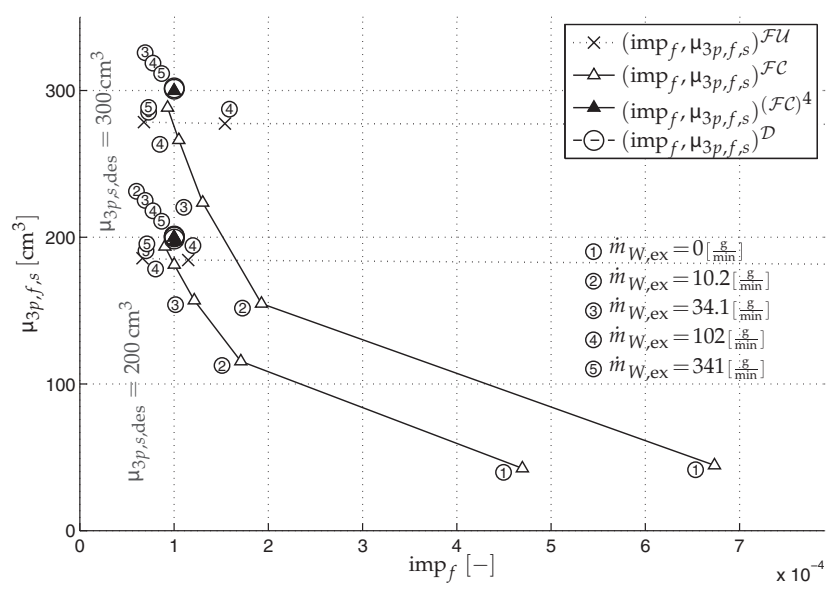

Abbildung 6: Wertepaare ( $\operatorname{imp}_{f}, \mu_{3 p, f, s}$ ) bei Anwendung der Vorgehensweisen aus Abschnitt 4.2 a) (FU), 4.2 b) (F्) , 4.2 c) $\left((\mathcal{F C})^{4}\right)$ sowie $4.3(\mathcal{D}) ;$ imp des $_{\text {de }}=10^{-4}$.

im verwendeten Rechenbeispiel ermittelt werden. Hier$\mathrm{zu}$ werden die in Abschnitt 4.1 für die oben genannten Kombinationen aus Mindestreinheiten und Produktmengen berechneten Temperaturprofile als Eingang des nicht idealisierten Modells (2), (4) verwendet. In Abbildung $5\left(\mathrm{imp}_{\mathrm{des}}=10^{-4}\right)$ bzw. $7\left(\mathrm{imp}_{\mathrm{des}}=10^{-6}\right)$ sind die resultierende Batchdauer $t_{f}$ und das resultierende dritte Moment der bevorzugten Enantiomere (gesamtes drittes Moment $\mu_{3 p, f}$ sowie drittes Moment der gewachsenen Saatkristalle $\mu_{3 p, f, s}$ ) als Wertepaare $(\cdot, \cdot)^{\mathcal{F U}}$ für $\mu_{3 p, s, \text { des }}=200 \mathrm{~cm}^{3}$ (links bzw. unten) sowie $\mu_{3 p, s, \text { des }}=$ $300 \mathrm{~cm}^{3}$ (rechts bzw. oben) eingezeichnet. In Abbildung 6 $\left(\mathrm{imp}_{\mathrm{des}}=10^{-4}\right)$ bzw. $8\left(\mathrm{imp}_{\mathrm{des}}=10^{-6}\right)$ sind entsprechende Wertepaare der resultierenden Verunreinigung $\operatorname{imp}_{f}$ und des resultierenden $\mu_{3 p, f, s}$ für $\mu_{3 p, s, \text { des }}=200 \mathrm{~cm}^{3}$ (unten) so-

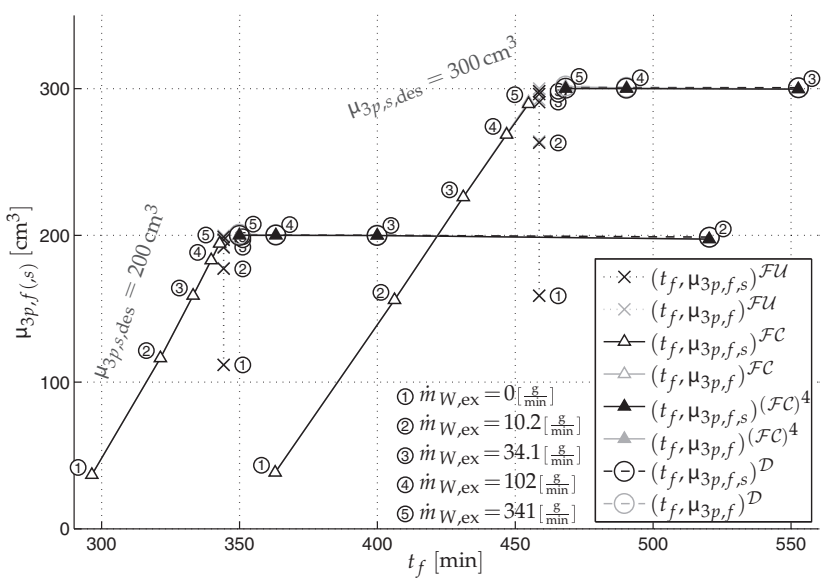

Abbildung 7: Ergebnisse wie in Abbildung 5, aber mit imp $\mathrm{pdes}=10^{-6}$.

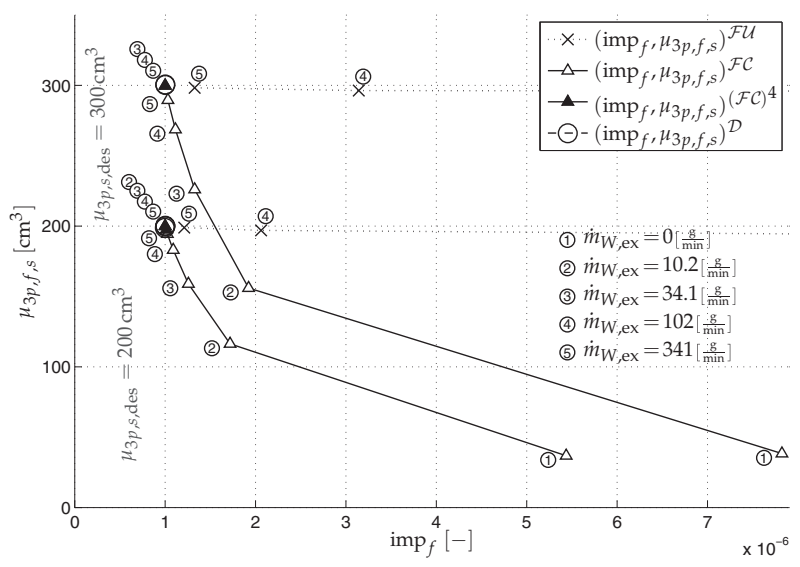

Abbildung 8: Ergebnisse wie in Abbildung 6, aber mit imp $\mathrm{p}_{\text {des }}=10^{-6}$.

wie $\mu_{3 p, s, \text { des }}=300 \mathrm{~cm}^{3}$ (oben) eingezeichnet. Man beachte, dass die Ergebnisse für die Flüssigkeitsaustauschraten $\dot{m}_{W, \text { ex }} \in\left\{0 \frac{\mathrm{g}}{\min }, 10.2 \frac{\mathrm{g}}{\min }, 34.1 \frac{\mathrm{g}}{\min }\right\}$ wegen zu großer $\operatorname{imp}_{f}$ Werte nicht in den Abbildungen 6 und 8 gezeigt werden. Man erkennt, dass die geforderte Produktmenge zwar bereits bei moderaten Austauschraten in guter Näherung erreicht wird, die geforderte Reinheit aber - außer bei sehr hohen Austauschraten - deutlich verfehlt wird. Daher wird im Folgenden eine korrigierte Vorgehensweise vorgeschlagen.

\section{b) Korrektur durch Inversion des nicht idealisierten Modells}

Der folgende heuristische Ansatz setzt sich aus zwei Schritten zusammen: Zunächst wird - wie in Abschnitt 4.1 beschrieben - die Optimierungsaufgabe für das idealisierte Modell gelöst. Die resultierende optimale $\mathrm{KGV} f_{c}^{* \mathrm{Z}}(L)=$ 
$f_{c}^{\mathcal{F}}(L)$ wird in einem zweiten Schritt als Vorgabe für die Inversion des nicht idealisierten Modells nach Abschnitt 3.1 verwendet. Das sich ergebende Temperaturprofil $T^{\mathcal{F C}}(t)$ bewirkt demzufolge bei Anwendung auf das nicht idealisierte Modell die exakte Realisierung der KGV $f_{c}^{* \mathbf{Z}}(L)=$ $f_{c}^{\mathcal{F}}(L)$ der Gegenenantiomere. Dies hat zur Folge, dass

$$
\begin{aligned}
\mu_{3 c, f}=\mu_{3 c}\left(\tau_{c, f}\right) & \leq \mu_{3 c, \mathrm{des}} \\
\tau_{c, f} & =\tau_{\mathrm{des}} .
\end{aligned}
$$

Man kann nun qualitativ argumentieren, dass durch den Flüssigkeitsaustausch (in Verbindung mit der Symmetrieannahme) die Übersättigungen und damit die Wachstumsraten für bevorzugtes Enantiomer und Gegenenantiomer sehr ähnliche Verläufe aufweisen und damit $\tau_{c, f}=$ $\int_{0}^{t_{f}} G_{c}(\cdot) d t=\tau_{\text {des }}$ und $\tau_{p, f}=\int_{0}^{t_{f}} G_{p}(\cdot) d t$ nah beieinander liegen. Transformiert man das Momentenmodell (2a) in die $\tau_{p}$-Zeit, so erhält man

$$
\frac{d \mu_{i p}}{d \tau_{p}}=i \mu_{(i-1) p}, i=1 \ldots 3, \frac{d \mu_{0 p}}{d \tau_{p}}=\frac{B_{p}(\cdot)}{G_{p}(\cdot)},
$$

bzw. für die durch Wachstum aus Saatkristallen entstehenden Anteile $\mu_{i p, s}$

$$
\frac{d \mu_{i p, s}}{d \tau_{p}}=i \mu_{(i-1) p, s}, i=1 \ldots 3, \quad \mu_{0 p, s}=\mu_{0 p, \text { seed }} .
$$

Daher gilt

$$
\begin{aligned}
\mu_{3 p, f, s}=\mu_{3 p, s}\left(\tau_{p, f}\right)= & \mu_{0 p \text {,seed }} \tau_{p, f}^{3}+3 \mu_{1 p, \text { seed }} \tau_{p, f}^{2} \\
& +3 \mu_{2 p \text {,seed }} \tau_{p, f}+\mu_{3 p, \text { seed }}
\end{aligned}
$$

Wegen $\tau_{p, f} \approx \tau_{\text {des }}$ wird die durch Wachstum der Saatkristalle entstehende Menge des bevorzugten Enantiomers in etwa der geforderten Menge entsprechen, d.h. $\mu_{3 p, f, s} \approx$ $\mu_{3 p, s, \mathrm{des}}$. Da im nicht idealisierten Fall durch die Nukleation des bevorzugten Enantiomers zusätzliche Produktmenge entsteht, wird die resultierende Gesamtproduktmenge $\mu_{3 p, f}$ nicht wesentlich unter der geforderten Menge $\mu_{3 p, s, \text { des }}$ liegen. Wegen imp $f=\mu_{3 c, f} /\left(\mu_{3 p, f}+\mu_{3 c, f}\right)$ wird die zulässige Verunreinigung $\operatorname{imp}_{\text {des }}$ nicht deutlich überschritten werden können.

Diese Sachverhalte bestätigen sich im vorgestellten Rechenbeispiel (siehe Abb. 5-8). Insbesondere kann man feststellen, dass die Abweichungen von der geforderten Mindestreinheit durch Einführung der diskutierten Korrektur deutlich geringer ausfallen. Man sieht, dass im Fall $\operatorname{imp}_{\text {des }}=10^{-4}$ das gesamte dritte Moment $\mu_{3 p, f}$ des bevorzugten Enantiomers durch den Nukleationsanteil er-

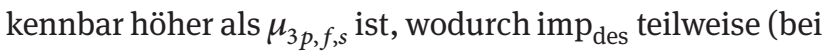

sehr hohen $\dot{m}_{W \text {,ex }}$ ) sogar unterschritten wird. Dahingegen hat die Spezifikation einer sehr geringen Verunreinigung $\left(\mathrm{imp}_{\mathrm{des}}=10^{-6}\right)$ hier den günstigen Nebeneffekt, dass auch die Nukleation des bevorzugten Enantiomers weitgehend unterdrückt wird, vergleiche Abbildungen 5 und 7. Abbildung 4 zeigt die korrigierten Temperaturprofile $T^{\mathcal{F}}(t)$, für $\mu_{3 p, s, \mathrm{des}}=200 \mathrm{~cm}^{3}$ und $\mathrm{imp}_{\mathrm{des}}=10^{-4}$ (unten) sowie $\mathrm{imp}_{\mathrm{des}}=10^{-6}$ (oben).

In der Implementierung der Inversion des nicht idealisierten Modells wird das Differenzialgleichungssystem (vgl. Abschnitt 3.1) in MATLAB ${ }^{\circledR}$ mit der Funktion ode 45 $\left(\right.$ RelTol $\left.=10^{-6}\right)$ gelöst. Die Berechnungszeiten für den Korrekturschritt lagen in etwa bei 20 Sekunden.

\section{c) Iteratives Vorgehen zur Einhaltung der Vorgaben}

Das in Abschnitt 4.2 b) beschriebene Verfahren hat den Nachteil, dass die in (23) definierten Nebenbedingungen nicht exakt (wenn auch genauer als bei Anwendung des unkorrigierten Temperaturprofils) eingehalten werden.

Um eine beliebig genaue Einhaltung $\mathrm{zu}$ erzwingen, wird das folgende iterative Vorgehen bei gegebenen $\mu_{3 p, s, \text { des }}$ und $\operatorname{imp}_{\mathrm{des}}$ vorgeschlagen:

$$
\begin{aligned}
1 . \quad I:=1, \quad \mu_{3 p, s, \mathrm{des}}^{(\mathcal{F C})^{I}} & =\mu_{3 p, s, \mathrm{des}}, \\
\mu_{3 c, \text { des }}^{(\mathcal{F C})^{I}} & =\mu_{3 p, s, \mathrm{des}} \cdot \frac{\mathrm{imp}_{\mathrm{des}}}{1-\mathrm{imp}_{\mathrm{des}}} .
\end{aligned}
$$

2. Bestimme $\tau_{\text {des }}^{(\mathcal{F C})^{I}}$ durch Lösen von (21b), mit $\mu_{3 p, s}\left(\tau_{\mathrm{des}}^{(\mathcal{F C})^{I}}\right)=\mu_{3 p, s, \mathrm{des}}^{(\mathcal{F C})^{I}}$.

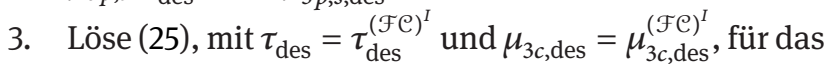
idealisierte Modell mit dem Verfahren in Abschnitt 4.1.

4. Führe den Korrekturschritt nach Abschnitt 4.2 b) durch die dynamische Inversion des nicht idealisierten Modells aus, bezeichne das resultierende $\mu_{3 p, f, s}$ als $\mu_{3 p, f, s}^{(\mathcal{F C})^{I}}$ und das resultierende $\mu_{3 p, f}$ als $\mu_{3 p, f}^{(\mathcal{F C})^{I}}$.

5. Aktualisiere die Vorgaben für den nächsten Iterationsschritt mittels eines Korrekturfaktors $\kappa^{I}$,

$$
\begin{aligned}
\kappa^{I} & =\mu_{3 p, s, \text { des }} \cdot\left(\mu_{3 p, f, s}^{(\mathcal{F C})^{I}}\right)^{-1}, \\
\mu_{3 p, s, \text { des }}^{(\mathcal{F C})^{I+1}} & =\mu_{3 p, s, \text { des }}^{(\mathcal{F C})^{I}} \cdot \kappa^{I}, \\
\mu_{3 c, \text { des }}^{(\mathcal{F C})^{I+1}} & =\mu_{3 p, f}^{(\mathcal{F C})^{I}} \cdot \frac{\operatorname{imp}_{\mathrm{des}}}{1-\operatorname{imp}_{\mathrm{des}}} \cdot \kappa^{I} .
\end{aligned}
$$

6. Abbrechen bei akzeptablem Fehler in den Nebenbedingungen in (23); sonst $I:=I+1$, fahre mit 2. fort.

Der erste Durchlauf entspricht also dem im vorigen Abschnitt 4.2 b) beschriebenen Verfahren. Das daraus resultierende dritte Moment $\mu_{3 p, f, s}^{(\mathcal{F C})^{I}}$ der gewachsenen Saatkristalle wird i. A. nicht der Vorgabe $\mu_{3 p, s, \text { des }}$ entsprechen, 


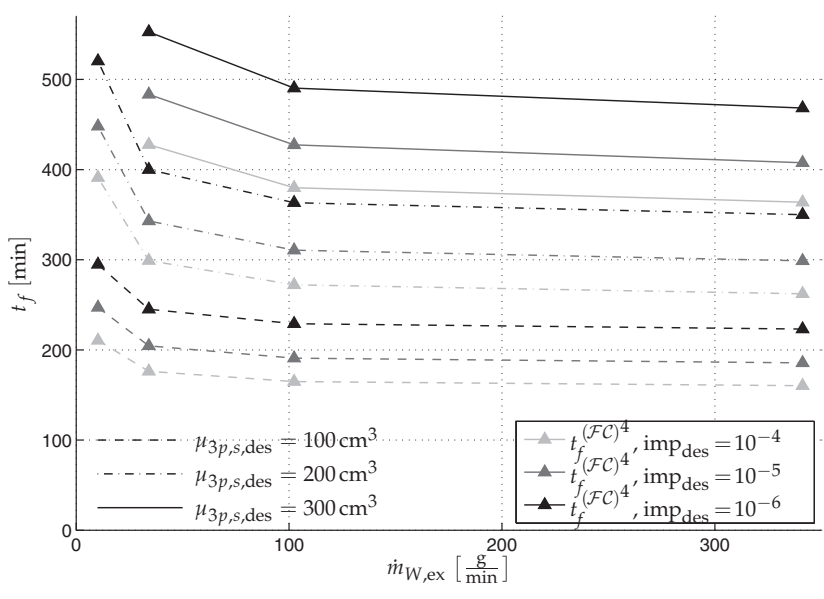

Abbildung 9: Batchdauer $t_{f}$ in Abhängigkeit von $\dot{m}_{W, \mathrm{ex}}$ bei Anwendung der Vorgehensweise nach Abschnitt $4.2 \mathrm{c}$ ) (4 Durchläufe). In Fällen mit zu geringen $\dot{m}_{W \text {,ex }}$ wurde keine Lösung gefunden.

weshalb mittels (34b) eine entsprechend nach oben (bzw. unten) korrigierte Vorgabe $\mu_{3 p, s, \text { des }}^{(\mathcal{F})^{I+1}}$ für die anschließende Wiederholung der idealisierungsbasierten Lösung angesetzt wird. Dabei wird unter zusätzlicher Beachtung des durch Nukleation des bevorzugten Enantiomers entstandenen dritten Moments mittels (34c) auch das gemäß (25) maximal zulässige dritte Moment der Gegenenantiomere korrigiert. Durch wiederholte Ausführung der Schritte 2. bis 6. nähert man sich dem ursprünglich geforderten $\mu_{3 p, s, \text { des }}$ unter Beachtung der maximal zulässigen Verunreinigung $i m p_{\text {des }}$ an.

Für das Rechenbeispiel konvergierte das vorgeschlagene Verfahren, und bereits nach 4 Iterationsschritten stellte sich die geforderte Genauigkeit ein. Die für $\operatorname{imp}_{\text {des }} \in\left\{10^{-4}, 10^{-6}\right\}$ und $\mu_{3 p, s, \text { des }} \in\left\{200 \mathrm{~cm}^{3}, 300 \mathrm{~cm}^{3}\right\}$ resultierenden Wertepaare $\left(t_{f}, \mu_{3 p, f, s}\right)$ bzw. $\left(t_{f}, \mu_{3 p, f}\right)$ und $\left(\operatorname{imp}_{f}, \mu_{3 p, f, s}\right)$ sind ebenfalls in den Abbildungen 5 bis 8 gezeigt und mit $(\mathcal{F C})^{4}$ bezeichnet. Die Einhaltung der Nebenbedingungen musste allerdings (insbesondere bei niedrigen Flüssigkeitsaustauschraten) mit einer merklichen Erhöhung der Batchdauer $t_{f}$ erkauft werden. Bei zu niedriger Flüssigkeitsaustauschrate konnte keine Lösung gefunden werden. Dies deutet darauf hin, dass dort die Vorgaben nicht erfüllbar sind.

Der Zusammenhang zwischen Flüssigkeitsaustauschrate $\dot{m}_{W \text {,ex }}$ und Batchdauer $t_{f}$ ist nochmals in Abbildungen 9 für verschiedene Vorgaben bzgl. Produktmenge $\mu_{3 p, s, \text { des }}$ und Reinheit imp des dargestellt. Daraus lassen sich wichtige Leistungsdaten des Prozesses ablesen. Durch Erhöhung des Flüssigkeitsaustauschs auf ein moderates Niveau kann die gewünschte Menge der bevor- zugten Enantiomersorten mit der geforderten Reinheit in merklich kürzerer Zeit hergestellt werden, wohingegen eine sehr hohe Austauschrate nur eine geringe weitere Verkürzung der Batchdauer bewirkt. Die Batchdauer $t_{f}$ steigt (zumindest in dem betrachteten Bereich) etwa linear mit der geforderten Produktmenge $\mu_{3 p, s, \text { des }}$ an. Dahingegen können bei moderater Verlängerung der Batchdauer wesentlich höhere Reinheiten erzielt werden.

Die Rechenzeiten für das gesamte Verfahren mit vier Iterationsschritten $\left((\mathcal{F} \mathcal{C})^{4}\right)$ lagen bei ca. 300 Sekunden.

\section{3 Überprüfung durch Optimierung eines diskreten Temperaturprofils}

Natürlich ist die in Abschnitt 4.1 und 4.2 beschriebene Vorgehensweise ein heuristisch geprägter suboptimaler Lösungsansatz. Im Folgenden soll deswegen mittels eines rechnerisch sehr viel aufwändigeren Ansatzes demonstriert werden, dass die gefundenen Lösungen in der Tat näherungsweise optimal sind. Dieser Ansatz basiert auf einer Parametrierung des Stellgrößenprofils des nicht idealisierten Modells und anschließender Lösung des resultierenden Parameteroptimierungsproblems. Insbesondere wird gezeigt, dass sich in der in Abschnitt 4.2 c) berechneten Batchdauer $t_{f}^{(\mathcal{F C})^{4}}$ bei Einhaltung der geforderten Reinheitsnebenbedingung imp $\mathrm{p}_{\text {des }}$ nur insignifikant mehr Produktmenge $\mu_{3 p, f, s}$ erzeugen lässt. Für das nicht idealisierte Modell soll also folgendes Optimierungsproblem gelöst werden:

$$
\max _{T(t)} \mu_{3 p, f, s} \text { so dass } \begin{aligned}
t_{f} & =t_{f}^{(\mathcal{F C})^{4}} \\
\operatorname{imp}_{f} & \leq \operatorname{imp}_{\mathrm{des}} .
\end{aligned}
$$

Man beachte, dass die in dieser Formulierung bekannte Zeitdauer die Lösung vereinfacht.

Zur Lösung von (35) wird durch Parametrierung des Stellgrößenprofils $T(t)$ mittels 15 äquidistanter Stützpunkte und linearer Interpolation ein Parameteroptimierungsproblem abgeleitet und gelöst. In der Implementierung wird dazu ein Gradientenverfahren verwendet, wobei die Reinheitsnebenbedingung durch Gradientenprojektion berücksichtigt wird (ähnlich wie dies in [4], Abschnitt 7.4, für die Optimierung kontinuierlicher Profile behandelt wird). Die Gradienten werden hier aber nicht mit Hilfe des adjungierten Systems ermittelt, sondern rechenintensiv durch finite Differenzen (Perturbation des Parametervektors) approximiert. Die notwendigen Simulationen werden in MATLAB ${ }^{\circledR}$ mit der Funktion ode45 $\left(\operatorname{RelTol}=10^{-6}\right)$ durchgeführt. Der initiale Parametervektor entspricht einer konstanten Temperatur bei der $\operatorname{imp}_{f}=\mathrm{imp}_{\text {des }}$. Die Schrittweite und die feste Iterationszahl (200) wurden im 
Hinblick auf zuverlässige Konvergenz (empirisch) adäquat eingestellt.

Die aus der numerischen Lösung des Problems (35) für das nicht idealisierte Modell resultierenden Wertepaare $\left(t_{f}, \mu_{3 p, f, s}\right)^{\mathcal{D}},\left(t_{f}, \mu_{3 p, f}\right)^{\mathcal{D}}$ und $\left(\mathrm{imp}_{f}, \mu_{3 p, f, s}\right)^{\mathcal{D}}$ sind in den Abbildungen 5 bis 8 eingetragen. In der Tat ist jeweils keine oder nur eine sehr geringe Steigerung von $\mu_{3 p, f, s}^{\mathcal{D}}$ gegenüber $\mu_{3 p, f, s}^{(\mathcal{F C})^{4}}$ erkennbar. Die mit der Vorgehensweise aus Abschnitt 4.2 c) erhaltenen Tupel $\left(t_{f}, \operatorname{imp}_{f}, \mu_{3 p, f, s}\right)^{(\mathcal{F C})^{4}}$ liegen demzufolge sehr nah am Optimum.

Die Berechnungszeiten für die Lösung des aus (35) resultierenden Parameteroptimierungsproblems sind ungefähr 20 mal größer als die für das iterative Vorgehen nach Abschnitt $4.2 \mathrm{c}$ ) benötigten Rechenzeiten.

\section{Zusammenfassung und Ausblick}

In diesem Beitrag wurde zunächst eine inversionsbasierte Steuerung für Modelle eines gekoppelten Batchprozesses der bevorzugten Kristallisation zwecks Realisierung gewünschter Kristallgrößenverteilungen vorgestellt. Es wurde der symmetrische Fall betrachtet, für den sich die Modellinversion auf einfachere Weise durchführen lässt. Bei weiteren idealisierenden Annahmen ergibt sich ein orbitell flaches Modell, für das die Inversion zu einem rein algebraischen Problem wird.

Im zweiten Teil des Aufsatzes wurde zunächst bei Vorgaben bezüglich Reinheit und Produktmenge eine zeitoptimale Steuerung für das idealisierte Modell bestimmt. Ein iterativer Algorithmus, bei dem diese Lösung durch Inversion des nicht idealisierten Systems korrigiert wird, liefert auch für das nicht idealisierte System eine fast zeit- optimale Steuerung bei beliebig genauer Einhaltung der Nebenbedingungen. Dies wurde für ein Rechenbeispiel demonstriert. Der vorgeschlagene Algorithmus ist dabei numerisch deutlich effizienter als ein auf Diskretisierung des Stellgrößenprofils beruhender Ansatz. Man beachte, dass die in Abschnitt 4.2 vorgestellte Vorgehensweise nur dann sinnvoll ist, wenn die Spezifikation hinsichtlich der Produktmenge sich auf die gewachsenen Saatkristalle bezieht. Andernfalls könnten Lösungen mit gezielt herbeigeführter Nukleation der bevorzugten Enantiomere optimal sein.

In der Zukunft sollen auch nicht symmetrische Verhältnisse behandelt werden, z.B. bei nicht identischen KGVen der Saatkristalle in den beiden Gefäßen. Möglicherweise lässt sich bei nicht zu großer Asymmetrie die hier vorgestellte Vorgehensweise unter Verwendung des Inversionsverfahrens [16] für den asymmetrischen Fall entsprechend erweitern. Größere Asymmetrien können jedoch auch durch die Optimierungsaufgabe selbst bedingt sein, z. B. bei unterschiedlichem Verwendungszweck der beiden hergestellten Enantiomersorten. Weiterhin ist die Berücksichtigung zusätzlicher Stellgrößen, insbesondere der Flüssigkeitsaustauschrate, zu prüfen. Außerdem soll der Einfluss der Anfangsbedingungen (z. B. die KGVen der Saatkristalle betreffend) untersucht bzw. optimiert werden. Schließlich sollen die entwickelten Steuerungsverfahren um eine geeignete Rückführung zur Kompensation von Unsicherheiten (z. B. die KGVen der Saatkristalle oder die Nukleationskinetiken betreffend) und Störungen ergänzt werden.

Eingang 18. Januar 2013.

\section{Literatur}

1. I. Angelov, J. Raisch, M. P. Elsner, and A. Seidel-Morgenstern. Optimal operation of enantioseparation by batch-wise preferential crystallization. Chemical Engineering Science, 63(5):1282-1292, 2008.

2. S. Antonov, A. Fehn, and A. Kugi. A new flatness-based control of lateral vehicle dynamics. Vehicle System Dynamics, 46(9):789-801, 2008.

3. S. A. Bhat and B. Huang. Preferential crystallization: Multiobjective optimization framework. AlChE Journal, 55(2):383395, Feb. 2009.

4. A. E. Bryson, Jr. and Y.-C. Ho. Applied Optimal Control. Hemisphere Publishing Corporation, 1975.

5. T. Y. Chiu and P. D. Christofides. Nonlinear control of particulate processes. AIChE Journal, 45(6):1279-1297, 1999.
6. S. Devasia, D. Chen, and B. Paden. Nonlinear inversion-based output tracking. IEEE Transactions on Automatic Control, 41(7):930-942, 1996.

7. M. P. Elsner, G. Ziomek, and A. Seidel-Morgenstern. Efficient separation of enantiomers by preferential crystallization in two coupled vessels. AIChE Journal, 55(3):640-649, Feb. 2009.

8. M. P. Elsner, G. Ziomek, and A. Seidel-Morgenstern. Simultaneous preferential crystallization in a coupled batch operation mode. Part II: Experimental study and model refinement. Chemical Engineering Science, 66(6):1269-1284, 2011.

9. M. Fliess, J. Lévine, P. Martin, and P. Rouchon. Design of trajectory stabilizing feedback for driftless flat systems. In Proceedings of the 3rd European Control Conference (ECC'95), pages 1882-1887, 1995. 
10. K. Graichen, V. Hagenmeyer, and M. Zeitz. A new approach to inversion-based feedforward control design for nonlinear systems. Automatica, 41(12):2033-2041, 2005.

11. K. Graichen, V. Hagenmeyer, and M. Zeitz. Feedforward control with online parameter estimation applied to the Chylla-Haase reactor benchmark. Journal of Process Control, 16(7):733-745, 2006.

12. M. Guay, S. Kansal, and J. F. Forbes. Trajectory optimization for flat dynamic systems. Ind. Eng. Chem. Res., 40(9):2089-2102, 2001.

13. S. Hofmann, M. J. Eicke, M. P. Elsner, and J. Raisch. Innovative control strategies for coupled preferential crystallization. In Proceedings of WCPT6 2010 - World Congress on Particle Technology, Apr. 2010.

14. S. Hofmann and J. Raisch. Application of optimal control theory to a batch crystallizer using orbital flatness. In 16th Nordic Process Control Workshop, pages 61-67. Nordic Working Group on Process Control, Aug. 2010.

15. S. Hofmann and J. Raisch. Solutions to inversion problems in preferential crystallization of enantiomers-part I: Batch crystallization in a single vessel. Chemical Engineering Science, 80:253-269, 2012.

16. S. Hofmann and J. Raisch. Solutions to inversion problems in preferential crystallization of enantiomers-Part II: Batch crystallization in two coupled vessels. Chemical Engineering Science, 88:48-68, 2013.

17. H. K. Khalil. Nonlinear Systems. Prentice Hall, 3rd edition, 2001.

18. T. Kleinert, M. Weickgenannt, B. Judat, and V. Hagenmeyer. Cascaded two-degree-of-freedom control of seeded batch crystallisations based on explicit system inversion. Journal of Process Control, 20(1):29-44, 2010.

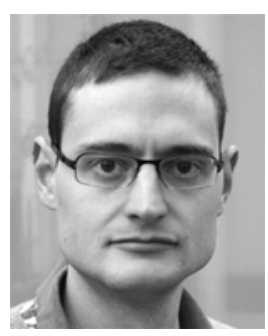

Dipl.-Ing. Steffen Hofmann

Fachgebiet Regelungssysteme, Sekretariat EN 11, Einsteinufer 17, D-10587 Berlin hofmann@control.tu-berlin.de

Dipl.-Ing. Steffen Hofmann hat Elektrotechnik und Informationstechnik an der TU Darmstadt studiert und ist seit 2008 wissenschaftlicher Mitarbeiter am Fachgebiet Regelungssysteme der TU Berlin. Hauptarbeitsgebiete: Regelung von Kristallisationsprozessen zur Enantiomertrennung.
19. Z. K. Nagy and R. D. Braatz. Robust nonlinear model predictive control of batch processes. AlChE Journal, 49(7):1776-1786, 2003.

20. D. Ramkrishna. Population Balances: Theory and Applications to Particulate Systems in Engineering. Academic Press, New York, 2nd edition, 2000.

21. A. D. Randolph and M. A. Larson. Theory of Particulate Processes. Academic Press, San Diego, 2nd edition, 1988.

22. W. Respondek. Orbital feedback linearization of single-input nonlinear control systems. In Proceedings of the 4th IFAC Nonlinear Control Systems Design Symposium (NOLCOS'98), pages 499-504, 1998.

23. R. Rothfuß, J. Rudolph, and M. Zeitz. Flachheit: Ein neuer Zugang zur Steuerung und Regelung nichtlinearer Systeme. atAutomatisierungstechnik, 45(11):517-525, 1997.

24. D. Sarkar, S. Rohani, and A. Jutan. Multi-objective optimization of seeded batch crystallization processes. Chemical Engineering Science, 61(16):5282-5295, 2006.

25. H. Sira-Ramírez and S. K. Agrawal. Differentially Flat Systems. Control Engineering Series. Marcel Dekker, Inc, 2004.

26. W. M. Sluis. A necessary condition for dynamic feedback linearization. Systems \& Control Letters, 21(4):277-283, 1993.

27. U. Vollmer and J. Raisch. Control of batch cooling crystallisers based on orbital flatness. International Journal of Control, 76(16):1635-1643, 2003.

28. U. Vollmer and J. Raisch. Control of batch crystallization a system inversion approach. Chem. Eng. Proc., 45:874-885, 2006.

29. X. Wang and C. B. Ching. A systematic approach for preferential crystallization of 4-hydroxy-2-pyrrolidone: Thermodynamics, kinetics, optimal operation and in-situ monitoring aspects. Chemical Engineering Science, 61:2406-2417, 2006.

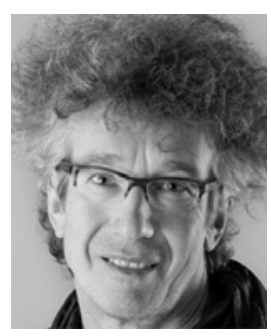

Prof. Dr.-Ing. Jörg Raisch

Fachgebiet Regelungssysteme, Sekretariat EN 11, Einsteinufer 17, D-10587 Berlin raisch@control.tu-berlin.de

Prof. Dr.-Ing. Jörg Raisch leitet das Fachgebiet Regelungssysteme an der Fakultät für Elektrotechnik und Informatik der TU Berlin. Er ist externes wissenschaftliches Mitglied des Max-Planck-Instituts für Dynamik komplexer technischer Systeme in Magdeburg und leitet dort die Fachgruppe System- und Regelungstheorie. Hauptarbeitsgebiete: Regelung hybrider Systeme, ereignisdiskrete Systeme, hierarchische Regelung, Regelung von Kristallisationsprozessen, medizintechnische Anwendungen. 